\title{
SUBREGIONAL ANALYSIS OF PREFRONTAL CORTEX MEDIATION OF COGNITIVE FUNCTIONS IN RATS, MONKEYS, AND HUMANS
}

\section{Preface}

The frontal lobes are widely viewed as the executive of the brain because of their role in strategic planning that governs the expression of cognitive and behavioral functions associated with other brain regions. In the highly developed brains of humans and nonhuman primates, the anterior portion of the frontal lobes - the prefrontal cortex (PFC) - has been identified as crucial to this essential process. In recent years, there has been progress in understanding the functional significance of the PFC, but, as is usually the case, answers to questions raise more questions. For example, to what extent is there regional specialization within the PFC, and what is the relationship between subregions in terms of organizing behavior? Are PFC subregions functionally organized in terms of domain or process specificity? Of the various cognitive and memory-related processes associated with the PFC, working memory (WM) is probably the most extensively studied. Nevertheless, important questions related, for example, to memory load and manipulation of information in WM still require answers. In addition, what is the role of the PFC in mediating attention, response selection, the use of rules and strategies, and the nature of executive control? These and other questions are addressed in the papers in the present issue that describe frontal lobe functions of higher species.

Fuster's paper, entitled "The Prefrontal Cortex of the Primate: A Synopsis" provides a broad contextual framework for several issues that are subsequently discussed by various authors from other perspectives. Fuster describes morphological and physiological features of the PFC and reviews functional connections with other brain regions that underscore the networks involved in the organization of goal-directed behavior. He argues that PFC-controlled systems are organized hierarchically, a point that is also made by others among the present authors. As well, his emphasis on the need to analyze WM into dissociable components provides the perfect transition to other papers in this issue.

Issues specifically related to WM are discussed in the next three papers. Postle and D'Esposito, in a paper entitled "Evaluating Models of the Topographical Organization of Working Memory Function in Frontal Cortex with Event-Related fMRI," and Owen, Lee, and Williams, in a paper entitled "Dissociating Aspects of Verbal Working Memory Within the Human Frontal Lobe: Further Evidence for a 'Process-Specific' Model of Lateral Frontal Organization," directly challenge the view that the PFC can be anatomically segregated into regions that selectively control spatial and nonspatial WM functions. Both groups provide evidence, based on fMRI studies, that the dorsolateral (DLPFC) and ventrolateral (VLPFC) regions of the PFC are organized according to processing demands of tasks rather than the types of information associated with the tasks. The evidence is consistent with Owen et al.'s review of the functional neuroimaging literature that provides numerous examples in which performance of spatial and visual WM tasks has been accompanied by activation of both the DLPFC and VLPFC areas. Baldo and Shimamura, in their article entitled "Spatial and Color Working Memory in Patients With Lateral Prefrontal Cortex Lesions," take a similar position, based on a neuropsychological investigation of WM performance in tasks involving spatial or nonspatial visual stimuli. Patients with damage to the PFC were generally impaired on both tasks, although there were differential effects related to delay and interference factors. Careful examination of the results did not reveal performance differences that could be related to differences in lesions along the dorsal-ventral dimension; rather, the evidence points to a multiple-components interpretation in which different tasks depend on shared processes that are mediated in dorsal and ventral regions of the PFC. 
Christoff and Gabrieli, in a thoughtful and comprehensive review paper entitled "The Frontopolar Cortex and Human Cognition: Evidence for a Rostrocaudal Hierarchical Organization Within the Human Prefrontal Cortex," extend theoretical notions regarding the functional organization of the PFC. These authors agree with the view (also expressed by Owen et al. and Postle \& D'Esposito) that the PFC is capable of mediating the retrieval of small amounts of information in WM, but that when the task requires manipulation and/or monitoring of information, the DLPFC is also recruited. In addition, Christoff and Gabrieli review evidence that there is functional organization within the PFC along the rostrocaudal dimension. They conclude that dorsolateral regions are specialized to process externally generated information, whereas the frontopolar cortex is selectively involved in dealing with internally generated or self-referential information. Further, in developing an elegant three-stage hierarchical model of PFC function, Christoff and Gabrieli identify important points of convergence between the clinical neuropsychological and functional neuroimaging literatures.

A somewhat different division of function for the DLPFC and VLPFC is presented by Rushworth, in the article entitled "Anatomical and Functional Subdivision Within the Primate Lateral Prefrontal Cortex." He proposes that on the basis of anatomical connections, one can envision that the DLPFC is primarily involved in mediating an action system rather than just spatial working memory, whereas the VLPFC is involved in mediating a sensory knowledge and identity system rather than just form WM. This represents a somewhat different view of subregional specificity of the PFC in comparison with the WM or depth-of-processing views.

Postle and D'Esposito and Baldo and Shimamura also address the issue of hemispheric differences in WM function. These investigators found no evidence of lateralized effects in their WM paradigms. However, as reviewed by Raye, Johnson, Mitchell, Nolde, and D'Esposito, in their paper entitled "fMRI Investigations of Left and Right PFC Contributions to Episodic Remembering," there is evidence of asymmetry in frontal lobe activation across a wide range of tasks and functions. Raye et al. report a series of fMRI studies in which they investigated left and right PFC activity during recognition memory, and the relationship between such activity and task complexity. Among several findings, the authors report that stimulus modality and processing effects correlated with hemisphere, but that, as task complexity increased demands on reflective operations, activation was more likely to be distributed across the two hemispheres. The authors interpreted this result in terms of their hemisphere interaction hypothesis, which holds that interhemispheric cooperation in task performance is related to the complexity of information-processing demands.

In another paper, entitled "The Role of the Prefrontal Cortex in Dynamic Filtering," Shimamura suggests that the key to understanding the PFC is based on the understanding of how PFC mediation of the executive system controls information processing. He proposes a dynamic filtering theory of PFC function based on executive control over selecting, maintaining, updating, and rerouting of information. These are general functions of the PFC with limited subregional specificity.

In rodents, Kesner, in a review article entitled "Subregional Analysis of Mnemonic Functions of the Prefrontal Cortex in the Rat," proposes that there are differential subregional (precentral cortex, anterior cingulate cortex, prelimbic and infralimbic as well as medial orbital cortices, agranular insular and lateral orbital cortices, and ventral orbital and ventrolateral orbital cortices) functional contributions to cognitive and memory function. It is proposed that the PFC in the rat can be fractionated in terms of functions associated with a slightly revised rule model that incorporates domain-specific and level-of-processing types of information. It is proposed that there is a hierarchy of lower order, higher order, and highest order rules that can support different subregions of the rat PFC.

In addition to the processing of mnemonic functions, the PFC may play a role in mediating attentional and response selection processes. Granon and Poucet, in the article entitled "Involvement of the Rat Prefrontal Cortex in Cognitive Functions: A Central Role for the Prelimbic Area," review the role of the prelimbic area and suggest that this area, which is assumed to be homologous to the lateral PFC, supports complex integrative functions incorporating attentional, WM, and response selection processes. The prelimbic area not only maintains information, but also is involved in higher order integration. In a further elaboration of the role of the pre- and infralimbic cortex function based on neurochemical modulation, in the article entitled "The Contribution of Cholinergic and Dopaminergic Afferents in the Rat Prefrontal Cortex to Learning, Memory, and Attention," Ragozzino presents a critical review of data that indicate that both dopamine and acetylcholine modulate WM and attentional processes within this region. Similarly, in the article entitled "Functional Dissociation Between Dorsal and Ventral Regions of the Medial Prefrontal Cortex in Rats," 
Gisquet-Verrier, Winocur, and Delatour present data supporting the idea that the pre- and infralimbic cortex is implicated in processes that support behavioral flexibility and attentional mechanisms, whereas the anterior cingulate cortex mediates temporal processes. Finally, in the article entitled "Mixed Attentional and Executive Deficits in Medial Frontal Cortex Lesioned Rats," Passetti, Humby, Everitt, and Robbins present data providing additional support for a role of the prelimbic and infralimbic area in mediating attention based on impaired accuracy in a five-choice reaction time task following pre- and infralimbic lesions.

From this set of articles, it appears that there are likely to be subregional differences in function within the prefrontal cortex of humans, primates, and rodents. However, the differentiation based on cognitive function among the different subregions has led to multiple hypotheses, including differential roles in WM, attentional processes, selection of responses, action, perceptual representation, temporal processing, executive control, inhibitory control, and rule-based learning and selection of strategies. Furthermore, these subregions are assumed to be organized hierarchically or in parallel to each other. It is hoped that the publication of this issue of Psychobiology will engender a strong interest in further clarifying the exact role(s) for specific subregions of the PFC in both animals and humans. 